\title{
Modelos relativistas de discos de polvo magnetizados en un espaciotiempo conformestático axialmente simétrico
}

\author{
Guillermo A. González
}

Escuela de Física, Universidad Industrial de Santander, Bucaramanga, Colombia

\begin{abstract}
Resumen
Se presenta una familia infinita de nuevas soluciones de las ecuaciones de Einstein-Maxwell para espaciotiempos conformestáticos axialmente simétricos. La familia de soluciones describe discos delgados de polvo cuya fuente material presenta una corriente de conducción superficial. Las soluciones se obtienen expresando la función métrica y el potencial magnético en términos de una función auxiliar que satisface la ecuación de Laplace, una propiedad característica de los espaciotiempos conformestáticos. Al introducir una discontinuidad finita en las primeras derivadas del tensor m'etrico, se obtienen soluciones con una singularidad de tipo función delta con soporte en la hipersuperficie $z=0$, describiendo discos infinitesimalmente delgados de extensión infinita. El tensor de momentumenergía superficial y la densidad de corriente superficial del disco se obtienen entonces utilizando el formalismo de las distribuciones tensoriales y se analiza su contenido físico. Se encuentra entonces que la densidad de energía se comporta bien en todas partes, que el tensor de momentum-energía satisface todas los condiciones de energía y que, aunque los discos son de extensión infinita, su masa total es finita. Adicionalmente, se encuentra que los escalares cuadráticos de curvatura y los invariantes electromagnéticos presentan un comportamiento regular, de tal manera que el espaciotiempo está libre de singularidades. Finalmente, con el fin de ilustrar el comportamiento de la familia de discos delgados, se considera el modelo correspondiente al primer miembro de la familia y se analiza gráficamente el comportamiento de la densidad superficial de energía y de la densidad superficial de corriente.
\end{abstract}

Palabras clave: Relatividad General, Soluciones exactas, Campos de Einstein-Maxwell.

Relativistic models of magnetized dust disks in an axially symmetric conformastatic spacetime

Abstract

An infinite family of new solutions of the Einstein-Maxwell equations for axially symmetric conformastatic spacetimes is presented. This family of solutions describe thin dust disks made of material sources with a surface conduction current. The solutions are obtained by expressing the metric function and the magnetic potential in terms of an auxiliary function which satisfies the Laplace equation, a characteristic property of the conformastatic spacetimes. By introducing then a finite discontinuity on the first derivatives of the metric tensor, solutions with a delta function type singularity with support on the hypersurface $z=0$ are obtained, describing so infinitesimally thin disks of infinite extension. Then, the surface energy-momentum and the surface current density of the disk are obtained by using the formalism of tensorial distributions and their physical content is analyzed. It was found that the energy density behaves well everywhere, that the energy-momentum tensor satisfies all the energy conditions and that, although the discs are of infinite extension, their total mass is finite. Furthermore, it is found that the curvature quadratic scalars and the electromagnetic invariants are regular everywhere, so that the spacetime is free of singularities. Finally, in order to illustrate the behavior of the family of thin disks, we consider the model for the first family member and graphically analyzes the behavior of the surface energy density and the surface current density.

Key words: General relativity, Exact solutions, Einstein-Maxwell fields.

Correspondencia:

Guillermo A. González, guillego@uis.edu.co, Recibido: 9 de marzo de 2014, Aceptado: 8 de octubre de 2014 


\section{Introducción}

Una de las características más fundamentales de un sistema aislado en el universo es la simetría axial, siendo ejemplos importantes de esta clase de configuraciones planetas, estrellas, galaxias, discos de acreción y agujeros negros. De acuerdo con esto, a través de los años se ha dedicado una gran cantidad de esfuerzo al estudio teórico del campo gravitacional generado por esta clase de fuentes aisladas, tanto en el marco de la teoría newtoniana de la gravitación como en el de la teoría general de la relatividad. Ahora bien, en el contexto de la relatividad general, la obtención de soluciones de las ecuaciones de Einstein que describan de manera auto-consistente los campos gravitacionales y las fuentes materiales que los generan es un problema de gran dificultad. Para obtener esta clase de soluciones se deben plantear y resolver simultáneamente el problema "exterior", un problema de contorno para las ecuaciones de Einstein en el vacío cuya solución determina el campo externo, y el problema "interior", cuya solución determina la estructura y la dinámica de la fuente en su propio campo gravitacional.

Por otro lado, cuando la fuente es un disco infinitesimalmente delgado, el problema interior se reduce a la formulación de condiciones de frontera para la solución exterior. Así entonces, se pueden obtener soluciones auto-consistentes resolviendo el problema exterior sujeto a condiciones de frontera apropiadas para obtener fuentes de materia con un comportamiento físicamente razonable. Además, a pesar de su naturaleza altamente ideal, los modelos relativistas de configuraciones discoidales infinitesimalmente delgadas de materia autogravitante presentan clara relevancia astrofísica puesto que pueden usarse para modelar discos de acreción, galaxias en equilibrio termodinámico y la superposición de galaxias y agujeros negros. En consecuencia, un buen número de trabajos se han dedicado a la obtención y análisis físico de soluciones exactas de las ecuaciones de Einstein correspondientes a este tipo de modelos. Así, varios autores han obtenido diferentes clases de soluciones exactas correspondientes a discos delgados estáti$\cos$ [1-13] y estacionarios [14-18].

Fuentes discoidales para un espaciotiempo estacionario axialmente simétrico con campos magnéticos son igualmente de importancia astrofísica principalmente en el estudio de estrellas de neutrones, enanas blancas y formación de galaxias. De a cuerdo con esto, esta clase de configuraciones se han estudiado extensivamente en la literatura, tanto desde el contexto puramente astrofísico como en relatividad general. Sin embargo, existen muy pocas soluciones exactas de las ecuaciones de Einstein-Maxwell correspondientes a esta clase de fuentes [19-25]. Ahora bien, una característica principal de las soluciones anteriormente mencionadas es el hecho de que el tensor de momentum-energía de la fuente describe un fluido anisótropo. De igual manera, casi todas estas soluciones, ex- cepto la presentada por Letelier en [20], corresponden a fuentes que no sólo son magnetizadas sino que presentan una densidad superficial de carga diferente de cero. Así entonces, la relevancia astrofísica de éstas no es clara debido a que no existe evidencia observacional de objetos astrofísicos cargados.

Teniendo en cuenta lo anterior, en este trabajo se presenta una familia infinita de discos de polvo axialmente simétricos cuya fuente material presenta una corriente de conducción superficial. Las soluciones se obtendrán mediante la técnica del "problema inverso" [26], en la cual se toma una solución de las ecuaciones de vacío de Einstein-Maxwell la cual presenta un discontinuidad en sus primeras derivadas a través del plano del disco y luego el tensor de momentum-energía y la densidad de corriente de la fuente se obtienen a partir de las ecuaciones de Einstein-Maxwell. Las propiedades físicas de la distribución de materia se estudian entonces analizando el tensor de momentum-energía y la densidad de corriente obtenidas. Con el fin de obtener estas soluciones discoidales, se resuelven las ecuaciones de vacío de Einstein-Maxwell para espaciotiempos conformestáticos axialmente simétricos; esto es, espaciotiempos estáticos en los cuales el elemento de línea espacial es conformemente plano [27,28] y en los cuales el elemento de línea se puede expresar en términos de una sola función métrica. Las soluciones se obtienen entonces expresando la función métrica y el potencial magnético en términos de una función auxiliar que satisface la ecuación de Laplace, una propiedad característica de los espaciotiempos conformestáticos [29]. Al introducir una discontinuidad finita en las primeras derivadas del tensor métrico, utilizando el procedimiento de "desplazamiento, corte y reflexión" [26], se obtienen soluciones con una singularidad de tipo función delta con soporte en la hipersuperficie $z=0$, describiendo discos infinitesimalmente delgados de extensión infinita.

El trabajo se encuentra organizado de la siguiente manera. Inicialmente, en la sección 2, se presentan las ecuaciones de Einstein-Maxwell para espaciotiempos conformestáticos y sus soluciones se encuentran resolviendo el sistema de ecuaciones de tal manera que las soluciones pueden expresarse en términos de una función solución de la ecuación de Laplace. Luego, en la sección 3, se obtienen el tensor de momentumenergía superficial del disco y la densidad de corriente superficial utilizando el formalismo de las distribuciones tensoriales. Se encuentran entonces las expresiones para la densidad de energía superficial del disco así como para su masa total y se muestra explícitamente que los discos están de acuerdo con todas las condiciones de energía. A continuación, en la sección 4, se presenta una familia de discos obtenida tomando para la ecuación de Laplace una familia de soluciones definida mediante una expansión en términos de polinomios de Legendre. Se obtienen entonces las expresiones explícitas para todas las variables que caracterizan el modelo y se analiza 
su comportamiento general. Se muestra entonces que, aunque los discos delgados son de extensión infinita, su masa total es finita. Adicionalmente, se encuentra que los escalares cuadráticos de curvatura y los invariantes electromagnéticos presentan un comportamiento regular, de tal manera que el espaciotiempo está libre de singularidades. Para ilustrar el comportamiento de la familia de discos delgados, se considera el modelo correspondiente al primer miembro de la familia y se analiza gráficamente el comportamiento de la densidad superficial de energía y de la densidad superficial de corriente. Finalmente, en la sección 5, se presenta una breve discusión de los resultados principales.

\section{Las ecuaciones de Einstein-Maxwell}

Las ecuaciones de Einstein-Maxwell, en unidades geométricas de Heaviside-Lorentz tales que $c=8 \pi G=\mu_{0}=$ $\epsilon_{0}=1$, toman la forma

$$
\begin{aligned}
R_{a b}-\frac{1}{2} g_{a b} R & =T_{a b}, \\
F_{; b}^{a b} & =0,
\end{aligned}
$$

con el tensor de momentum-energía dado por

$$
T_{a b}=F_{a c} F_{b}^{c}-\frac{1}{4} g_{a b} F_{c d} F^{c d},
$$

donde

$$
F_{a b}=A_{b, a}-A_{a, b}
$$

es el tensor de campo electromagnético y $A_{a}$ es el potencial electromagnético.

Ahora bien, para un espacio-tiempo conformestático axialmente simétrico, el elemento de línea se puede expresar como [27]

$$
d s^{2}=-e^{2 \psi} d t^{2}+e^{-2 \psi}\left(r^{2} d \varphi^{2}+d r^{2}+d z^{2}\right)
$$

donde $x^{a}=(t, \varphi, r, z)$ son las coordenadas cilíndricas usuales y la función métrica $\psi$ sólo depende de $r$ y $z$. Así, tomando el potencial electromagnético como

$$
A_{a}=(0, A, 0,0),
$$

y suponiendo que el potencial magnético $A$ igualmente sólo depende de $r$ y $z$, las componentes no nulas del tensor de campo electromagnético son

$$
\begin{aligned}
& F_{\varphi r}=-A_{, r}, \\
& F_{\varphi z}=-A_{, z},
\end{aligned}
$$

de modo que se tiene un campo magnético puro, axialmente simétrico.

Considerando entonces la métrica dada por (5) y el potencial electromagnético como (6), las ecuaciones de EinsteinMaxwell en el vacío son equivalentes al sistema

$$
\begin{aligned}
& r\left[\left(r^{-1} A_{, r}\right)_{, r}+\left(r^{-1} A_{, z}\right)_{, z}\right]+2 \nabla A \cdot \nabla \psi=0, \\
& 2 r^{2} \psi_{, r} \psi_{, z}+e^{2 \psi} A_{, r} A_{, z}=0, \\
& 2 r^{2} \psi_{, r}^{2}=e^{2 \psi} A_{, z}^{2} \\
& 2 r^{2} \psi_{, z}^{2}=e^{2 \psi} A_{, r}^{2} \\
& \nabla^{2} \psi=\nabla \psi \cdot \nabla \psi
\end{aligned}
$$

donde $\nabla$ es el operador diferencial usual en coordenadas cilíndricas para una función axialmente simétrica. Así, de las ecuaciones (10) - (12) es fácil ver que $A$ y $\psi$ están relacionadas mediante el sistema sobre-determinado

$$
\begin{aligned}
& A_{, r}= \pm \sqrt{2} r e^{-\psi} \psi_{, z}, \\
& A_{, z}=\mp \sqrt{2} r e^{-\psi} \psi_{, r},
\end{aligned}
$$

cuya condición de integrabilidad está garantizada cuando $\psi$ es una solución de la ecuación (13), y la ecuación (9) se satisface trivialmente.

Con el fin de resolver la ecuación (13), ésta se escribe primero en la forma equivalente

$$
\nabla^{2}\left(e^{-\psi}\right)=0
$$

y entonces se expresa la función métrica $\psi$ mediante la relación

$$
e^{-\psi}=1-U
$$

de tal manera que $U(r, z)$ es una solución axialmente simétrica de la ecuación de Laplace,

$$
\nabla^{2} U=0
$$

la cual debe anularse en el infinito con el fin de tener un espacio-tiempo asintoticamente plano. Adicionalmente, si sólo se consideran soluciones que sean negativas en todas partes, $e^{\psi}$ será en todas partes positiva y no singular. Así entonces, en términos de $U(r, z)$, las ecuaciones (14) y (15) toman la forma

$$
\begin{aligned}
& A_{, r}= \pm \sqrt{2} r U_{, z}, \\
& A_{, z}=\mp \sqrt{2} r U_{, r},
\end{aligned}
$$


un sistema sobre-determinado cuya condición de integrabilidad está garantizada por la ecuación de Laplace (18). De acuerdo con esto, sólo es necesario resolver la ecuación de Laplace para obtener la función métrica $\psi$ y el potencial magnético $A$ se obtiene resolviendo el sistema sobre-determinado anterior.

Ahora bien, soluciones correspondientes a distribuciones discoidales delgadas deben ser funciones simétricas de $z$ con sus primeras derivadas con respecto a $z$ discontinuas en $z=0$ [26]. Por lo tanto, con el fin de obtener esta clase de soluciones, se supondrá que $U(r, z)$ es una función continua en todas partes y con derivadas continuas de tal manera que, resolviendo el sistema sobre-determinado (19) - (20), se obtendrá un potencial magnético $A(r, z)$ igualmente continuo en todas partes y con derivadas continuas. Utilizando entonces el procedimiento de "desplazamiento, corte y refelxión" [26], después de tener la solución completa para las ecuaciones de Einstein-Maxwell se hará la transformación $z \rightarrow|z|+a$, en donde $a$ es una constante positiva arbitraria, de tal manera que se obtendrán soluciones continuas en todas partes pero con las primeras derivadas con respecto a $z$ discontinuas en la superficie del disco. El resultado será una solución con una singularidad del tipo función delta sobre $z=0$, la cual se puede interpretar como un disco delgado infinito.

\section{Contenido físico de la fuente}

Como se señaló en la sección anterior, las soluciones de las ecuaciones de Einstein-Maxwell correspondientes a fuentes discoidales son funciones pares de la coordenada $z$, continuas en todas partes pero con sus primeras derivadas con respecto a $z$ discontinuas en el disco. Por lo tanto, el contenido material y electromagnético del disco será descrito por funciones las cuales son distribuciones con soporte sobre el disco. De acuerdo con esto, para obtener el tensor de momentumenergía y la densidad de corriente de la fuente, se expresa el salto a través del disco de las primeras derivadas con respecto a $z$ del tensor métrico como

$$
\gamma_{a b}=\left[g_{a b, z}\right]=\left.2 g_{a b, z}\right|_{z=0^{+}},
$$

y el salto a través del disco del tensor campo electromagnético como

$$
\left[F_{z a}\right]=\left[A_{a, z}\right]=\left.2 A_{a, z}\right|_{z=0^{+}},
$$

donde se ha hecho uso de la simetría de reflexión con respecto a $z=0$.

Usando entonces la formulación en términos de distribuciones tensoriales [30-32] o las condiciones de juntura sobre la curvatura extrínseca de cascarones delgados [33-35], se puede escribir el tensor métrico como

$$
g_{a b}=g_{a b}^{+} \theta(z)+g_{a b}^{-}\{1-\theta(z)\}
$$

de tal manera que el tensor de Ricci se expresa como

$$
R_{a b}=R_{a b}^{+} \theta(z)+R_{a b}^{-}\{1-\theta(z)\}+H_{a b} \delta(z),
$$

donde $\theta(z)$ y $\delta(z)$ son, respectivamente, las distribuciones de Heaviside y Dirac con soporte en $z=0$. En la expresión anterior los tensores $g_{a b}^{ \pm}$y $R_{a b}^{ \pm}$son el tensor métrico y el tensor de Ricci en las regiones $z \geq 0$ y $z \leq 0$, respectivamente, mientras que

$$
H_{a b}=\frac{1}{2}\left\{\gamma_{a}^{z} \delta_{b}^{z}+\gamma_{b}^{z} \delta_{a}^{z}-\gamma_{c}^{c} \delta_{a}^{z} \delta_{b}^{z}-g^{z z} \gamma_{a b}\right\}
$$

donde todas las cantidades están evaluadas en $z=0^{+}$.

De las ecuaciones de Einstein-Maxwell se obtiene entonces un tensor de momentum-energía de la forma

$$
T_{a b}=T_{a b}^{+} \theta(z)+T_{a b}^{-}\{1-\theta(z)\}+Q_{a b} \delta(z),
$$

y una densidad de corriente de la forma

$$
J^{a}=I^{a} \delta(z)
$$

donde los tensores $T_{ \pm}^{a b}$ son los tensores de momentum-energía electromagnéticos definidos mediante la ecuación (3) para las regiones $z \geq 0$ and $z \leq 0$, respectivamente, mientras que

$$
\begin{aligned}
Q_{a b}= & \frac{1}{2}\left\{\gamma_{a}^{z} \delta_{b}^{z}+\delta_{a}^{z} \gamma_{b}^{z}-\gamma^{z z} \delta_{b}^{a}-g^{z z} \gamma_{a b}\right. \\
& \left.+\gamma_{c}^{c}\left(g^{z z} g_{a b}-\delta_{a}^{z} \delta_{b}^{z}\right)\right\}
\end{aligned}
$$

es la contribución de la fuente discoidal al tensor de momentum-energía y

$$
I^{a}=\left[F^{a z}\right]
$$

es la contribución de la fuente discoidal a la densidad de corriente.

El tensor de momentum-energía superficial del disco (SEMT), $S_{a b}$, y la densidad de corriente superficial, $j^{a}$, se pueden obtener mediante las relaciones

$$
\begin{aligned}
S_{a b} & =\int Q_{a b} \delta(z) d s_{n}=e^{-\psi} Q_{a b}, \\
j^{a} & =\int I^{a} \delta(z) d s_{n}=e^{-\psi} I^{a},
\end{aligned}
$$

donde $d s_{n}=\sqrt{g_{z z}} d z$ es la medida física de longitud en dirección normal al disco. Por lo tanto, la única componente no nula de $S_{a b}$ es

$$
S_{00}=4 e^{3 \psi} \psi_{, z},
$$

y la única componente no nula de la densidad superficial de corriente $j^{a}$ es

$$
j^{1}=\frac{2 e^{3 \psi} A_{, z}}{r^{2}},
$$

donde todas las cantidades están evaluadas en $z=0^{+}$. 
Introduciendo la tétrada ortonormal de un observador estático,

$$
\begin{aligned}
e_{\overparen{t}}^{a} & =V^{a}=e^{-\psi} \delta_{0}^{a}, \\
e_{\overparen{\varphi}}^{a} & =W^{a}=r^{-1} e^{\psi} \delta_{1}^{a}, \\
e_{\widehat{r}}^{a} & =X^{a}=e^{\psi} \delta_{2}^{a}, \\
e_{\overparen{z}}^{a} & =Y^{a}=e^{\psi} \delta_{3}^{a},
\end{aligned}
$$

el SEMT se puede escribir como

$$
S^{a b}=\epsilon V^{a} V^{b} .
$$

De acuerdo con esto, la fuente de materia es un disco de polvo con una densidad superficial de energía dada por

$$
\epsilon=4 e^{\psi} \psi_{, z}
$$

y cuyo vector velocidad está dado por $V^{a}$, el vector temporal de la tétrada. La masa total del disco está dada entonces por la expresión

$$
M=2 \pi \int_{0}^{\infty} e^{-\psi} \epsilon r d r,
$$

obtenida usando la formula de Komar [35,36].

Por lo tanto, debido a que se tiene una fuente de polvo, todas las condiciones de energía [37] se reducen al requerimiento de que la densidad de energía sea mayor o igual que cero,

$$
\epsilon \geq 0
$$

Por otro lado, utilizando la ecuación (17), la densidad de energía se puede escribir como

$$
\epsilon=\frac{4 U_{, z}}{(1-U)^{2}}
$$

Adicionalmente, dado que $U(r, z)$ es una solución de la ecuación de Laplace, se puede considerar como el potencial newtoniano de una fuente con una densidad superficial de masa dada por

$$
\sigma=\frac{U_{, z}}{2 \pi}
$$

Así entonces, si la densidad de masa newtoniana $\sigma$ es mayor o igual que cero en todas partes, la densidad de energía relativista correspondiente será mayor o igual que cero en todas partes y no singular, teniendo en cuenta que sólo se consideran soluciones de la ecuación de Laplace negativas en todas partes.

De igual manera, la densidad superficial de corriente se puede escribir como

$$
j^{a}=j W^{a},
$$

de modo que el disco posee una densidad superficial de corriente de conducción en la dirección acimutal dada por

$$
j=\frac{2 e^{2 \psi} A_{, z}}{r},
$$

la cual, en términos de $U(r, z)$, se puede escribir como

$$
j=\mp \frac{2 U_{, r}}{(1-U)^{2}},
$$

y el signo se escoge de acuerdo con el signo escogido en el sistema sobre-determinado (19) - (20).

Ahora bien, las componentes del campo magnético medidas por el observador estático están dadas por

$$
\begin{aligned}
& B_{\widehat{r}}=\frac{e^{2 \psi} A_{, z}}{r}, \\
& B_{\widehat{z}}=-\frac{e^{2 \psi} A_{, r}}{r},
\end{aligned}
$$

mientras que $B_{\widehat{\varphi}}=0$. Por otro lado, las componentes de la aceleración del observador con respecto a la tétrada ortonormal son

$$
\begin{aligned}
& a_{\widehat{r}}=e^{\psi} \psi_{, r}, \\
& a_{\widehat{z}}=e^{\psi} \psi_{, r},
\end{aligned}
$$

así que, utilizando las ecuaciones (14) y (15), se obtiene

$$
\begin{aligned}
& B_{\widehat{r}}=\mp a_{\widehat{r}}, \\
& B_{\widehat{z}}=\mp a_{\widehat{z}},
\end{aligned}
$$

y así el campo gravitacional y el campo magnético están alineados, una propiedad característica de todo solución de las ecuaciones de Einstein-Maxwell en espaciotiempos conformestáticos [29]. Las líneas de campo se pueden obtener resolviendo la ecuación diferencial

$$
\frac{d z}{B_{\widehat{z}}}=\frac{d r}{B_{\widehat{r}}},
$$

la cual, utilizando las ecuaciones (47) y (48), se puede escribir como

$$
A_{, r} d r+A_{, z} d z=0 .
$$

De acuerdo con esto, la ecuación

$$
A(r, z)=k,
$$

con $k$ constante, se puede usar para determinar las líneas tanto del campo magnético como del campo gravitacional. 
Finalmente, con el fin de determinar la posible presencia de singularidades, consideraremos los escalares cuadráticos de curvatura [38]

$$
\begin{aligned}
\mathcal{K}_{I} & =R^{a b c d} R_{a b c d}, \\
\mathcal{K}_{I I} & =\frac{\epsilon^{a b}{ }_{k l} R^{k l c d} R_{a b c d}}{\sqrt{-g}}, \\
\mathcal{K}_{I I I} & =\frac{\epsilon^{a b k l} \epsilon^{c d m n} R_{k l m n} R_{a b c d}}{g},
\end{aligned}
$$

así como los invariantes electromagnéticos

$$
\begin{aligned}
\mathcal{F}_{I} & =F_{a b} F^{a b}, \\
\mathcal{F}_{I I} & =\frac{\epsilon^{a b c d} F_{a b} F_{c d}}{\sqrt{-g}},
\end{aligned}
$$

donde $\epsilon^{a b c d}$ es el símbolo de Levi-Civita y $g=\operatorname{det} g_{a b}$.

Utilizando entonces la solución (17), los escalares de curvatura se pueden escribir como

$$
\begin{aligned}
& \mathcal{K}_{I}=\frac{8 N_{1}}{(1-U)^{8}}+\frac{16 N_{2}}{(1-U)^{7}} \frac{U_{, r}}{r}+\frac{16}{(1-U)^{6}} \frac{U_{, r}^{2}}{r^{2}}, \\
& \mathcal{K}_{I I}=0 \\
& \mathcal{K}_{I I I}=4 \mathcal{K}_{I}-\frac{64|\nabla U|^{4}}{(1-U)^{8}}
\end{aligned}
$$

donde

$$
\begin{aligned}
N_{1}= & 7|\nabla U|^{4}+2(1-U)^{2}\left(U_{, r r}^{2}+U_{, z z}^{2}\right) \\
& +6(1-U)\left[U_{, r r}\left(U_{, r}^{2}-U_{, z}^{2}\right)+2 U_{, r} U_{, z} U_{, r z}\right], \\
N_{2}= & U_{, r r}(1-U)-3 U_{, z}^{2} .
\end{aligned}
$$

De igual manera, utilizando (7), (8), (19) y (20), los invariantes electromagnéticos se pueden escribir como

$$
\begin{aligned}
\mathcal{F}_{I} & =\frac{4|\nabla U|^{2}}{(1-U)^{4}}, \\
\mathcal{F}_{I I} & =0 .
\end{aligned}
$$

Así, el comportamiento de los escalares de curvatura y de los invariantes electromagnéticos está determinado por la función $U(r, z)$.

\section{Una familia de discos magnetizados}

Consideremos ahora una familia de soluciones de las ecuaciones de Einstein-Maxwell obtenida tomando para la ecuación de Laplace (18) una familia de soluciones definida mediante la expansión

$$
\mathcal{U}_{m}(r, z)=-\sum_{n=0}^{m} C_{n} \frac{P_{n}(z / R)}{R^{n+1}},
$$

para cada $m \geq 0$, donde $R^{2}=r^{2}+z^{2}, P_{n}(z / R)$ son los polinomios de Legendre usuales y las $C_{n}$ son constantes arbitrarias. Usando entonces para lo polinomios de Legendre la representación [39]

$$
P_{n}(z / R)=(-1)^{n} \frac{R^{n+1}}{n !} \frac{\partial^{n}}{\partial z^{n}}\left[\frac{1}{R}\right],
$$

se puede resolver fácilmente el sistema sobre-determinado (19) - (20), obteniendo para el potencial electromagnético la familia de soluciones

$$
\mathcal{A}_{m}(r, z)=\sum_{n=0}^{m}(-1)^{n} \frac{C_{n}}{n !} \frac{\partial^{n}}{\partial z^{n}}\left[\frac{z}{R}\right]
$$

tomando el signo negativo en (19), el signo positivo en (20), y la constante de integración igual a cero.

Así entonces, después de tener la solución definida mediante las ecuaciones (69) y (70), se hace la transformación $z \rightarrow|z|+a$ para obtener la solución discoidal correspondiente,

$$
\begin{aligned}
& U_{m}(r, z)=\mathcal{U}_{m}(r,|z|+a), \\
& A_{m}(r, z)=\mathcal{A}_{m}(r,|z|+a) .
\end{aligned}
$$

Adicionalmente, para satisfacer los requerimientos impuestos sobre $U(r, z)$ con el fin de tener un espacio-tiempo asintóticamente plano y satisfacer las condiciones de energía, las constantes $C_{n}$ se escogerán de tal manera que

$$
\begin{aligned}
U_{m}(r, z) & \leq 0, \\
U_{m, r}(r, z) & \geq 0, \\
U_{m, z}(r, z) & \geq 0, \\
\lim _{r \rightarrow \infty} U_{m}(r, z) & =0, \\
\lim _{z \rightarrow \infty} U_{m}(r, z) & =0,
\end{aligned}
$$


y así $U(r, z)$ será una función monótonamente creciente en $r$ y $z$, con un mínimo negativo en el origen.

Con $U(r, z)$ dado por (69), se pueden calcular fácilmente la densidad superficial de energía mediante la ecuación (42) y la densidad superficial de corriente de conducción mediante la ecuación (46), las cuales se pueden expresar como

$$
\begin{aligned}
\epsilon_{m}(r) & =\frac{\partial}{\partial a}\left[\frac{4}{1-U_{m}(r, 0)}\right], \\
j_{m}(r) & =\frac{\partial}{\partial r}\left[\frac{2}{1-U_{m}(r, 0)}\right] .
\end{aligned}
$$

Así, utilizando las expresión explícita para $U_{m}(r, z)$, las expresiones anterores se pueden escribir convenientemente como

$$
\begin{aligned}
\epsilon_{m}(r) & =\frac{4 \widetilde{\epsilon}_{m}(\widetilde{r})}{a}, \\
j_{m}(r) & =\frac{2 \widetilde{j}_{m}(\widetilde{r})}{a},
\end{aligned}
$$

con

$$
\widetilde{\epsilon}_{m}=\frac{\sum_{n=0}^{m}\left[(n+1) \widetilde{C}_{n} P_{n+1}\left(1 / \widetilde{R}_{0}\right)\right] / \widetilde{R}_{0}^{n+2}}{\left[1+\sum_{n=0}^{m} \widetilde{C}_{n} P_{n}\left(1 / \widetilde{R}_{0}\right) / \widetilde{R}_{0}^{n+1}\right]^{2}},
$$

y

$$
\widetilde{j}_{m}=\frac{\widetilde{r} \sum_{n=0}^{m}\left[\widetilde{C}_{n} P_{n+1}^{\prime}\left(1 / \widetilde{R}_{0}\right)\right] / \widetilde{R}_{0}^{n+3}}{\left[1+\sum_{n=0}^{m} \widetilde{C}_{n} P_{n}\left(1 / \widetilde{R}_{0}\right) / \widetilde{R}_{0}^{n+1}\right]^{2}},
$$

donde $\widetilde{r}=r / a, \widetilde{R}_{0}^{2}=1+\widetilde{r}^{2}$ y $\widetilde{C}_{n}=C_{n} / a^{n+1}$.

De las expresiones anteriores, teniendo en cuenta las condiciones (71) y el comportamiento de los polinomios de Legendre, se puede ver que la densidad de energía es siempre positiva, presenta su valor máximo en el centro del disco y se anula en el infinito. De igual manera, se puede ver que la densidad superficial de corriente se anula en el centro del disco, cuando $r=0$, presenta un máximo para un valor de $r \neq 0$ y decae después hasta anularse en el infinito. Adicionalmente, utilizando la expresión (74) para la densidad de energía, la integral para la masa total del disco se puede expresar como

$$
\widetilde{M}_{m}=\int_{1}^{\infty} \widetilde{\mu}_{m} \widetilde{R}_{0} d \widetilde{R}_{0}
$$

$\operatorname{con} \widetilde{M}_{m}=M_{m} / a \mathrm{y}$

$$
\widetilde{\mu}_{m}=\frac{\sum_{n=0}^{m}\left[(n+1) \widetilde{C}_{n} P_{n+1}\left(1 / \widetilde{R}_{0}\right)\right] / \widetilde{R}_{0}^{n+2}}{1+\sum_{n=0}^{m} \widetilde{C}_{n} P_{n}\left(1 / \widetilde{R}_{0}\right) / \widetilde{R}_{0}^{n+1}},
$$

de donde es fácil ver que

$$
\lim _{\widetilde{R}_{0} \rightarrow \infty} \frac{\widetilde{\mu}_{m+1}}{\widetilde{\mu}_{m}}=1 .
$$

De acuerdo con esto, $y$ teniendo en cuenta el teorema de comparación del límite [40], la integral para la masa total del modelo $m+1$ será convergente si la integral para el modelo $m$ converge. Así entonces, si la masa total del disco para el modelo $m=0$ es finita, entonces la masa total del disco para cualquier valor de $m>0$ será finita también. Finalmente, usando la representación (67) para los polinomios de Legendre, es fácil ver que

$$
\frac{U_{m, r}}{r}=\sum_{n=0}^{m} \frac{(-1)^{n} C_{n}}{n !} \frac{\partial^{n}}{\partial z^{n}}\left[\frac{1}{R^{3}}\right],
$$

una expresión regular para to $r$ y $z$. Así entonces, el comportamiento regular de esta expresión y las condiciones (71) garantizan el comportamiento regular de los escalares cuadráticos de curvatura y de los invariantes electromagnéticos, de tal manera que el espaciotiempo definido por la solución (69) está libre de singularidades.

Con el fin de ilustrar el comportamiento de la familia de discos delgados, consideremos el modelo correspondiente al primer miembro de la familia, $m=0$, en el cual las variables físicas del modelo están dadas por

$$
\begin{aligned}
U_{0} & =-\frac{\widetilde{C}_{0}}{\sqrt{\widetilde{r}^{2}+(1+|\widetilde{z}|)^{2}}}, \\
\widetilde{A}_{0} & =\frac{\widetilde{C}_{0}(1+|\widetilde{z}|)}{\sqrt{\widetilde{r}^{2}+(1+|\widetilde{z}|)^{2}}}, \\
\widetilde{\epsilon}_{0} & =\frac{\widetilde{C}_{0}}{\widetilde{R}_{0}\left[\widetilde{C}_{0}+\widetilde{R}_{0}\right]^{2}}, \\
\widetilde{j}_{0} & =\frac{\widetilde{C}_{0} \widetilde{r}}{\widetilde{R}_{0}\left[\widetilde{C}_{0}+\widetilde{R}_{0}\right]^{2}}, \\
\widetilde{M}_{0} & =\ln \left(1+\widetilde{C}_{0}\right),
\end{aligned}
$$

donde, como en las expresiones anteriores, $\widetilde{A}=A / a$. Como se puede ver, la masa del modelo $m=0$ es finita, lo cual garantiza que la masa total del disco en todos los modelos para $m>0$ es finita también.

En la figura 1 se muestran las curvas de nivel de la función métrica $U(r, z)$ para el modelo $m=0$, escaladas al valor de la constante $\widetilde{C}_{0}$. Así, $U_{0} / \widetilde{C}_{0}=-1$ en el origen y el valor de $U_{0} / \widetilde{C}_{0}$ en cada curva de nivel aumenta incrementalmente en valores de 0,05 . Igualmente, en la figura 1 se muestran también las curvas de nivel del potencial magnético $\widetilde{A}_{0}$ escaladas 

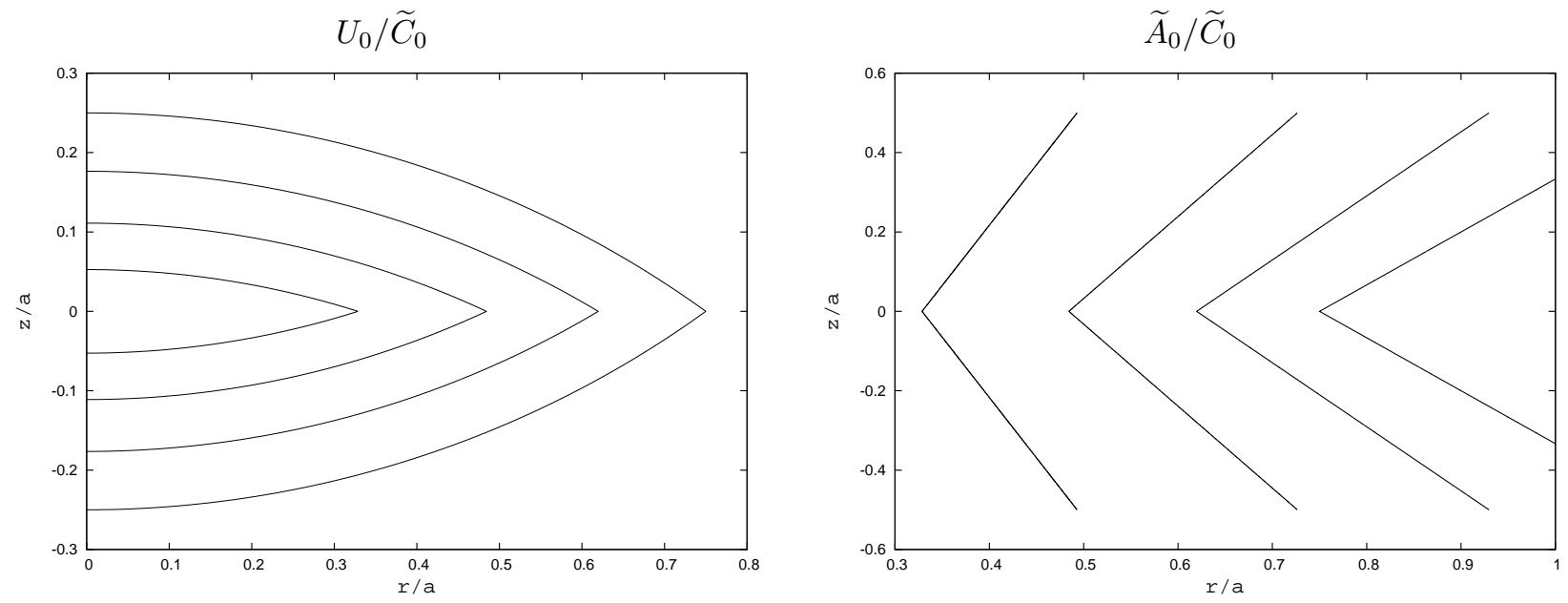

Figura 1: Curvas de nivel de la función métrica $U_{0}$ escaladas al valor de la constante $\widetilde{C}_{0}$. Así, $U_{0} / \widetilde{C}_{0}=-1$ en el origen y el valor de $U_{0} / \widetilde{C}_{0}$ en cada curva de nivel aumenta incrementalmente en valores de 0,05 . Se muestran también las curvas de nivel del potencial magnético $\widetilde{A}_{0}$ escaladas al valor de la constante $\widetilde{C}_{0}$. Así, $\widetilde{A}_{0} / \widetilde{C}_{0}=1$ en el origen y el valor de $U_{0} / \widetilde{C}_{0}$ en cada curva de nivel aumenta incrementalmente en valores de 0,05 .

$\widetilde{\epsilon}$

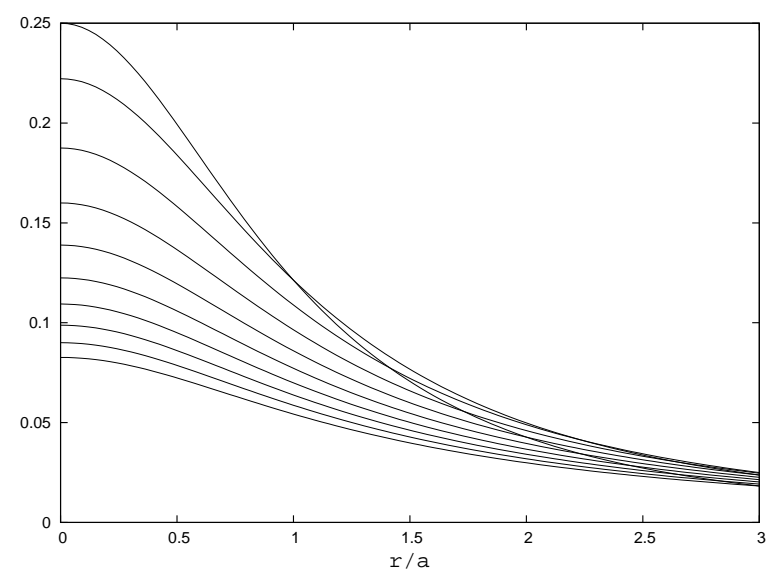

$\widetilde{j}$

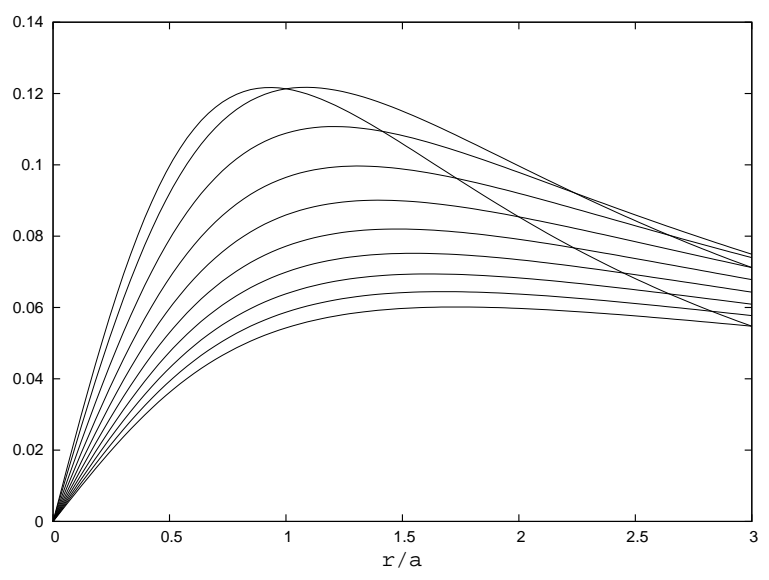

Figura 2: Densidad superficial de energía $\widetilde{\epsilon}$ como función de $\widetilde{r}$ para $\widetilde{C}_{0}=1$ (curva superior), .., 10 (curva inferior). la densidad de energía es siempre positiva y se anula en el infinito, presentando su valor máximo en el centro del disco. Se muestra también la densidad superficial de corriente $\widetilde{j}$ como función de $\widetilde{r}$ para $\widetilde{C}_{0}=1$ (curva superior), .., 10 (curva inferior). La densidad superficial de corriente se anula en el centro del disco, presentando un máximo para un valor de $\widetilde{r} \neq 0$ y decayendo después hasta anularse en el infinito. 
al valor de la constante $\widetilde{C}_{0}$. Así, $\widetilde{A}_{0} / \widetilde{C}_{0}=1$ en el origen y el valor de $U_{0} / \widetilde{C}_{0}$ en cada curva de nivel aumenta incrementalmente en valores de 0,05 . Posteriormente, en la figura 2, se muestra la densidad superficial de energía $\widetilde{\epsilon}$ como función de $\widetilde{r}$ para $\widetilde{C}_{0}=1$, correspondiente a la curva superior, hasta $\widetilde{C}_{0}=10$, correspondiente a la curva inferior. De igual manera, se muestra también en la figura 2 la densidad superficial de corriente $\widetilde{j}$ como función de $\widetilde{r}$ para $\widetilde{C}_{0}=1$, correspondiente a la curva superior, hasta $\widetilde{C}_{0}=10$, correspondiente a la curva inferior. Como se puede ver, la densidad de energía es siempre positiva y se anula en el infinito, presentando su valor máximo en el centro del disco, tal como se había mencionado anteriormente. Se puede ver igualmente que la densidad superficial de corriente se anula en el centro del disco, presentando un máximo para un valor de $\widetilde{r} \neq 0$ y decayendo después hasta anularse en el infinito, como también se había mencionado anteriormente.

\section{Conclusiones}

Se presentó una familia infinita de discos de polvo axialmente simétricos cuya fuente material presenta una corriente de conducción superficial. Los discos se obtuvieron resolviendo las ecuaciones de vacío de Einstein-Maxwell para un espaciotiempo conformestático axialmente simétrico, expresando la función métrica y el potencial magnético en términos de una función auxiliar que satisface la ecuación de Laplace. Ahora bien, la aparente contradicción entre la presencia de corriente superficial y la ausencia de presiones, radiales o tangenciales, es consecuencia directa del procedimiento utilizado para la obtención de las soluciones, mediante la técnica del "problema inverso" y el procedimiento de "desplazamiento, corte y reflexión" [26]. Sin embargo, a pesar de esta aparente contradicción, las soluciones son consistentes con el sistema completo de ecuaciones de Einstein-Maxwell para distribuciones de materia infinitesimalmente delgadas. Por otro lado, a pesar de que las soluciones presentadas son estáticas y así su relevancia astrofísica puede no ser muy alta, la obtención de soluciones estáticas se puede considerar como el primer paso para la obtención de soluciones estacionarias más realistas.

Analizando las propiedades de la distribución de materia, se encontró que la densidad de energía de los discos es siempre positiva y se anula en el infinito, presentando su valor máximo en el centro del disco. Así, dado que los discos tienen una fuente material de polvo, los modelos están en completo acuerdo con todas las condiciones de energía, un hecho de especial relevancia en el estudio de modelos relativistas de discos delgados. Además, aunque los discos son de extensión infinita, su masa total es finita. De igual manera, la densidad superficial de corriente se anula en el centro del disco, presentando pos- teriormente un máximo y decayendo después hasta anularse en el infinito. Adicionalmente, se encontró que los escalares cuadráticos de curvatura y los invariantes electromagnéticos presentan un comportamiento regular, de tal manera que el espaciotiempo está libre de singularidades. Ahora bien, aunque sólo se mostraron explícitamente las cantidades físicas correspondientes al primer miembro de la familia, cuando $m=0$, se puede mostrar fácilmente que todos los miembros de la familia para $m>0$ presentan un comportamiento similar.

\section{Agradecimientos}

El autor agradece el apoyo financiero de la Vicerrectoría de Investigación y Extensión de la Universidad Industrial de Santander a través el proyecto 5189.

\section{Bibliografía}

[1] W. A. Bonnor and A. Sackfield, Comm. Math. Phys. 8, 338 (1968)

[2] T. Morgan and L. Morgan, Phys. Rev. 183, 1097 (1969)

[3] L. Morgan and T. Morgan, Phys. Rev. D 2, 2756 (1970)

[4] B. H. Voorhees, Phys. Rev. D 5, 2413 (1972)

[5] D. Lynden-Bell and S. Pineault, Mon. Not. R. Astron. Soc. 185, 679 (1978)

[6] A. Chamorro, R. Gregory, and J. M. Stewart, Proc. R. Soc. London A413, 251 (1987)

[7] P. S. Letelier and S. R. Oliveira, J. Math. Phys. 28, 165 (1987)

[8] J. P. S. Lemos, Class. Quantum Grav. 6, 1219 (1989)

[9] J. Bičák, D. Lynden-Bell, and J. Katz, Phys. Rev. D 47, 4334 (1993)

[10] J. Bičák, D. Lynden-Bell, and C. Pichon, Mon. Not. R. Astron. Soc. 265, 126 (1993)

[11] G. A. González and P. S. Letelier, Class. Quantum Grav. 16, 479 (1999)

[12] G. A. González and O. A. Espitia, Phys. Rev. D 68, 104028 (2003)

[13] G. A. González, A. C. Gutiérrez-Piñerez and V. M. Viña-Cervantes. Phys. Rev. D 79, 124048 (2009)

[14] D. Lynden-Bell and S. Pineault, Mon. Not. R. Astron. Soc. 185, 695 (1978) 
[15] J. Bičák and T. Ledvinka, Phys. Rev. Lett. 71, 1669 (1993)

[16] C. Pichon and D. Lynden-Bell, Mon. Not. R. Astron. Soc. 280, 1007 (1996)

[17] G. A. González and P. S. Letelier, Phys. Rev. D 62, 064025 (2000)

[18] G. A. González, Revista Integración 20, 13 (2002)

[19] T. Ledvinka, J. Bičák, and M. Žofka, in Proceeding of 8th Marcel-Grossmann Meeting in General Relativity, edited by T. Piran (World Scientific, Singapore, 1999)

[20] P. S. Letelier, Phys. Rev. D 60, 104042 (1999)

[21] J. Katz, J. Bičák, and D. Lynden-Bell, Class. Quantum Grav. 16, 4023 (1999)

[22] G. García R. and G. A. González, Phys. Rev. D 69, 124002 (2004)

[23] G. García-Reyes and G. A. González, Class. Quantum Grav. 21, 4845 (2004)

[24] G. García-Reyes and G. A. González, Phys. Rev. D 70, 104005 (2004)

[25] G. García-Reyes and G. A. González, Braz. J. Phys. 37, 1094 (2007)

[26] G. A. González and A. C. Gutiérrez-Piñeres, Classical and Quantum Gravity 29, 135001 (2012).

[27] J. L. Synge, Relativity, the General Theory (NorthHolland Publishing Company, Amsterdam, 1966).
[28] H. Stephani, D. Kramer, M. MacCallum, C. Hoenselaers and E. Herlt, Exact Solutions of Einstein's Field Equations. (Cambridge University Press, 2003).

[29] G. A. González and R. Vera, Classical and Quantum Gravity 28, 025008 (2011).

[30] A. Papapetrou and A. Hamouni, Ann. Inst. Henri Poincaré 9, 179 (1968).

[31] A. Lichnerowicz, C.R. Acad. Sci. 273, 528 (1971).

[32] A. H. Taub, J. Math. Phys. 21, 1423 (1980).

[33] E. Israel, Nuovo Cimento 44B, 1 (1966).

[34] E. Israel, Nuovo Cimento 48B, 463 (1967).

[35] E. Poisson, A Relativist's Toolkit: The Mathematics of Black-Hole Mechanics. (Cambridge University Press, 2004).

[36] R. M. Wald, General Relativity. (The University of Chicago Press, 1984).

[37] S. W. Hawking and G. F. R. Ellis, The Large Scale Structure of Space-Time. (Cambridge University Press, 1973).

[38] C. Cherubini, D. Bini, S. Capozziello, and R. Ruffini, Int. J. Mod. Phys. D 11, 827 (2002).

[39] G. B. Arfken and H. J. Weber, Mathematical Methods for Physicists, 6th Edition, (Elsevier Academic Press, 2005).

[40] T. M. Apostol, Calculus, 2d Edition, (Wiley, 1967). 